\title{
Metallicity gradients of galaxies in the Herschel Reference Survey
}

\author{
Thomas M. Hughes
}

Astronomical Observatory, Ghent University, Belgium

email: thomas. hughes@ugent. be

\begin{abstract}
We introduce a pilot project to measure metallicitiy gradients for a sample of twenty nearby galaxies drawn from the Herschel Reference Survey (HRS), representative of normal, star-forming spiral galaxies. We have obtained optical spectroscopic observations using the Very Large Telescope with the FORS2 instrument in multi-object mode, targeting individual HiI and star-forming regions across the galaxy discs (P. I.: L. Cortese). From the ratios of the strong emission lines, we estimate the local gas-phase oxygen abundance and construct metallicity gradients. Combining these new data with Herschel PACS/SPIRE far-infrared photometric observations and Hi $21 \mathrm{~cm}$ line maps, to trace the cold dust and gas respectively, will allow the study of the relationships between stars, gas, dust and metals on sub-kiloparsec scales.
\end{abstract}

Keywords. cosmology: observations galaxies: spiral galaxies: evolution

\section{Preliminary reduction of VLT/FORS2 observations}

In Fig. 1, we show two example spectra from slits covering Hit regions in NGC 5669 (aka HRS 319; Boselli et al. 2010), extracted with the ESO Reflex pipeline. Using emission lines observed in each spectra at each position in our twenty galaxies, we apply the method of Hughes et al. (2013) and calibrations of Kewley \& Ellison (2008) to estimate gas-phase oxygen abundance gradients. We will then investigate, amongst other things, how strong metallicity gradients may bias global metallicity estimates derived from the integrated spectra of gas-stripped galaxies (see Hughes et al. 2013; Boselli et al. 2013).
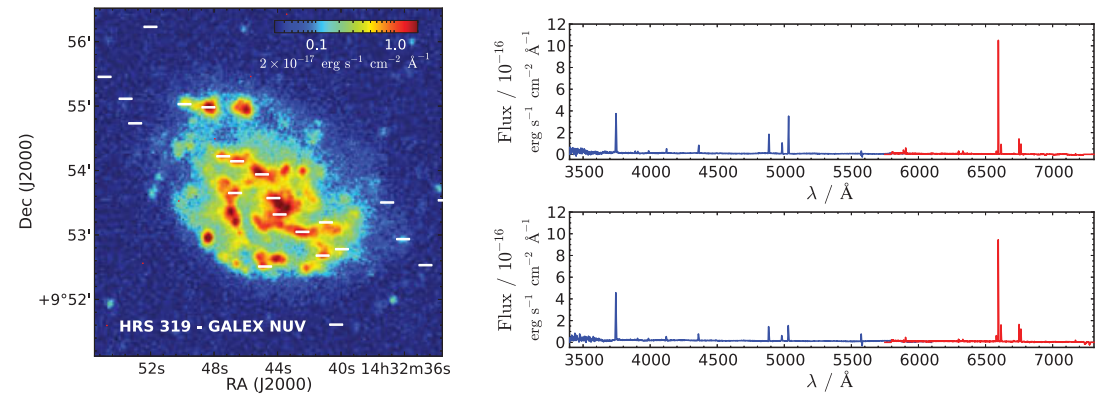

Figure 1. FORS2 MXU slit positions (white rectangles) covering HRS 319's HiI regions, traced here via $N U V$ emission (left), are presented alongside two example optical spectra extracted from the observations using the ESO Reflex standard data reduction pipeline (right).

\section{References}

Boselli, A., Eales, S., Cortese, L., Bendo, G., \& Chanial, P. et al.. 2010, PASP, 122, 261

Boselli, A., Hughes, T. M., Cortese, L., Gavazzi, G., \& Buat, V., 2013, A\&AA, 550, 114

Hughes, T. M., Cortese, L., Boselli, A., Gavazzi, G., \& Davies, J. I., 2013, A\&\&A, 550, 115

Kewley, L. \& Ellison, S., 2008, ApJ, 681, 1183 